\title{
MMP Simulation of Plasmonic Particles on Substrate Under E- Beam Illumination
}

\section{Book Chapter}

\section{Author(s):}

Koch, Ueli (iD; Niegemann, Jens; Hafner, Christian; Leuthold, Juerg (iD

Publication date:

2018

Permanent link:

https://doi.org/10.3929/ethz-b-000276048

Rights / license:

In Copyright - Non-Commercial Use Permitted

Originally published in:

Springer Series on Atomic, Optical, and Plasma Physics 99, https://doi.org/10.1007/978-3-319-74890-0_6 


\title{
MMP Simulation of Plasmonic Particles on Substrate under E-Beam Illumination
}

\author{
Ueli Koch, Jens Niegemann, Christian Hafner and Juerg Leuthold
}

\begin{abstract}
A novel numerical approach to investigate the resonance behavior of plasmonic particles on a substrate under e-beam illumination is presented. The method is based on the Multiple Multipole Program (MMP), a generalized point matching technique, and is augmented by the ability to compute layered media and electron energy loss spectroscopy (EELS) measurements. Furthermore, the whole framework is complemented by a mesh-based method that automatically places the multipole expansions and matching points for arbitrary three-dimensional geometries. The performance of our technique is analyzed by a series of numerical experiments. The EELS responses of a plasmonic split-ring resonator in free space and a plasmonic disk dimer on a membrane, as well as the resonant modes, are simulated. Finally, our implementation is compared to the established discontinuous Galerkin time domain (DGTD) method with respect to its computational efficiency. We show significantly improved performance especially for the computation of EELS resonance maps.
\end{abstract}

Ueli Koch

Institute of Electromagnetic Fields, Department of Information Technology and Electrical Engineering, ETH Zurich, Gloriastrasse 35, 8092 Zurich, Switzerland, e-mail: uelikoch @ethz.ch

Jens Niegemann

Lumerical Computational Solutions Inc., 1700-1095 West Pender Street, Vancouver, BC, Canada, e-mail: jniegemann@lumerical.com

Christian Hafner

Institute of Electromagnetic Fields, Department of Information Technology and Electrical Engineering, ETH Zurich, Gloriastrasse 35, 8092 Zurich, Switzerland, e-mail: hafner@ethz.ch

Juerg Leuthold

Institute of Electromagnetic Fields, Department of Information Technology and Electrical Engineering, ETH Zurich, Gloriastrasse 35, 8092 Zurich, Switzerland, e-mail: leuthold@ethz.ch 


\section{Introduction}

With the progress in nanofabrication over the past decades, metallic nanostructures with feature sizes well below $100 \mathrm{~nm}$ can today be produced routinely and reliably. This ability to structure metals on the nanoscale was instrumental in enabling the field of plasmonics, where the interaction between light and such tailored metallic nanostructures is studied and exploited for a variety of applications (e.g., see [1] and references therein). Despite the tremendous progress in the field, plasmonic nanostructures still present a significant challenge for both experimental and numerical characterization. Since the size of the particles is often well below the diffraction limit, one typically has to rely on electron microscopy to image the structures under investigation. A particularly interesting technique in this regard is to employ scanning transmission electron microscopy (STEM) in conjunction with electron energy loss spectroscopy (EELS) [2]. In this approach, fast electrons are passing nearby or through the plasmonic structure. Due to their local electric field, the electrons can excite plasmonic modes of the system and therefore lose a corresponding amount of kinetic energy. By performing spectroscopy on the transmitted electrons, one can therefore locally measure the excitation spectrum of the plasmonic structure. By raster-scanning over the sample, one can obtain EELS maps, showing such a spectrum at every point in a plane. One advantage of EELS over optical spectroscopy lies in its much better spatial resolution which is not limited by the diffraction of light. In addition, EELS allows to excite resonances that are optically dark due to their vanishing electric dipole moments.

In order to better understand experimental measurements and to properly interpret the EELS results, reliable EELS simulations play an important role. Traditionally, either the Finite Element Method (FEM) [3] or the Boundary Element Method (BEM) [4] were used to model EELS experiments. More recently, other numerical methods such as the Discrete Dipole Approximation (DDA) [5] and the Discontinuous Galerkin Time-Domain (DGTD) [6] approach were also shown to be suitable methods.

Since the computation of EELS maps requires a large number of calculations with identical geometry but different excitations (electron beams at different positions), numerical methods with multiple right-hand-sides features (multiple excitations) are favorable. The Multiple Multipole Program (MMP) [7, 8] supported multiple right-hand-sides already in early implementations. The latest implementation of MMP contained in the OpenMaXwell package [9] contains many additional advanced features for modeling and efficiently solving 2D and 3D electrodynamic problems, including eigenvalue problems (computation of resonators and of guided waves) and scattering problems in presence of strongly dispersive materials such as metals at optical frequencies. MMP provides built-in error estimates and a fast convergence if the interfaces of all objects are sufficiently smooth. As a consequence, MMP is very attractive for plasmonics and for EELS simulations in particular.

In the following, we will describe how to perform realistic EELS simulations with MMP. To this end, we first propose a novel method to automatically place the multipole expansions and matching points for arbitrary three-dimensional geome- 
tries. Since the dielectric properties of the substrate strongly influence the measurements, we also give details of how to properly handle substrates and stratified media in the MMP context. Finally, we provide the explicit expressions to include a relativistic electron beam as a source of excitation. To demonstrate the efficiency of our novel MMP approach, we perform a series of numerical experiments and compare the performance of our implementation to the established DGTD method.

\section{Generic MMP Simulation}

Although MMP is a flexible and powerful method for electromagnetic simulations, it also has some disadvantages. Probably the biggest inconveniences are the manual selection and placement of expansions and matching points. Even for an experienced user, this step can be time consuming when modeling complex three-dimensional geometries. To mitigate this problem, we have developed a novel generic approach which places the expansions and matching points automatically. Similarly to the classical boundary element method, it is based on a surface mesh. In the following, we will focus on triangular meshes, but our method is readily extended to any kind of surface discretization.

For a given mesh, we first compute the center $\mathbf{c}_{E}$ and the outward pointing normal vector $\mathbf{n}_{E}$ of each surface element $E$. Then, we place our expansions $e$ at a distance $d$ both inwards and outwards from the center, so the positions are

$$
\begin{aligned}
\mathbf{r}_{e, \text { in }} & =\mathbf{c}_{E}+d \mathbf{n}_{E}, \\
\mathbf{r}_{e, \text { out }} & =\mathbf{c}_{E}-d \mathbf{n}_{E} .
\end{aligned}
$$

The distance $d$ still remains to be defined. A first idea would be to choose $d$ based on the area of the respective element. However, we found that this approach can lead to poor conditioning and therefore reduces the robustness of our method. Instead, we employ an even simpler choice for $d$, namely a constant for the entire mesh. As we will demonstrate in Sect. 5.2.4, a distance of approximately $90 \%$ of the minimal curvature radius of the geometry usually produces good results while still remaining numerically stable.

In a second step, the matching points and their weights need to generated. For this, an idea from the finite element method is adapted. Since the error is distributed over the entire surface, it makes sense to use a two-dimensional quadrature rule on the surface elements. Generally, any order of quadrature rule could be chosen, but we found that only a second order rule provides a good balance between accuracy and computational effort (see Sect. 5.2.2). The locations of the matching points are exactly on the quadrature nodes. The corresponding weights for all boundary conditions of a single matching point $(m)$ are then given by $w_{m}=\sqrt{A_{E} w_{\text {quad }}}$, where $A_{E}$ and $w_{\text {quad }}$ are the element area and the quadrature weight, respectively.

With this simple procedure, the user only needs to provide a surface mesh and a distance parameter $d$ instead of spending a lot of time on the three-dimensional 
MMP modeling. To illustrate this generic process, we model a basic sphere. The surface mesh and the resulting distribution of expansions and matching points are shown in Fig. 1. We should mention that a sphere could probably be modeled more
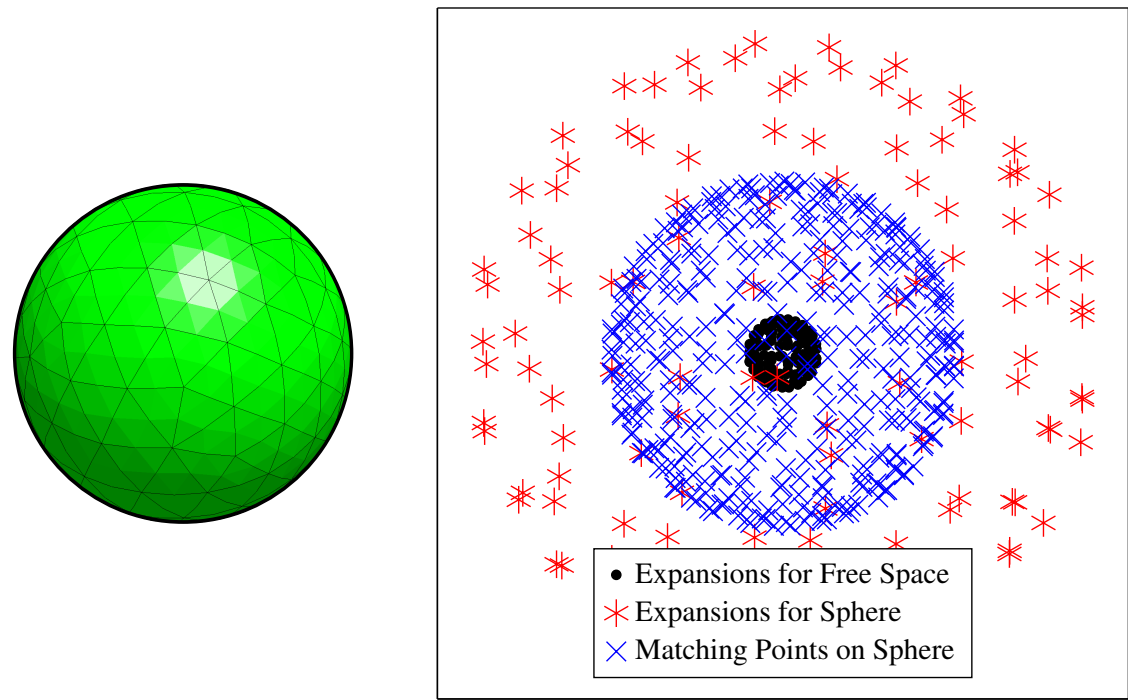

Fig. 1 Surface mesh with curvilinear elements generated by Netgen [10] and the generated distribution of the expansions and matching points for a floating sphere.

efficiently by manually placing higher order spherical expansions in the center. However, for more complex geometries the placement is usually not as obvious and a manual placement becomes time-consuming and inefficient.

\section{Dipoles in Layered Media}

The generic approach presented in the previous section allows us to efficiently model complex three-dimensional geometries with MMP. However, it does not make sense to also use this method to discretize infinitely extended structures such as substrates or membranes. We therefore need an alternative way to incorporate the effect of a substrate into our calculations. At this point, the flexibility of MMP with respect to the expansions come into play. Instead of using standard dipoles (or higher-order multipoles), we can also employ layered dipoles which directly incorporate the effect of the substrate into the basis functions. The electromagnetic fields caused by dipoles in stratified media are a well-studied subject [11, 12, 13]. We took advantage of this knowledge and implemented the layered dipole expansion in MMP [14] which saves us from explicitly discretizing the boundaries of layers and therefore reduces the memory requirements and the computational cost signifi- 
cantly. Since the computation of such a layered dipole expansion is fairly involved, we now present the most important steps in more detail.

\subsection{Layered Media}

In general, we follow the procedure and notation used in [11, Ch. 2] with some minor deviations. The basic idea consists of writing the field as an infinite superposition of plane waves,

$$
\begin{aligned}
\mathbf{E} & =\mathbf{E}_{0} \mathrm{e}^{\mathrm{i} \mathbf{k} \cdot \mathbf{r}}, \\
\mathbf{H} & =\mathbf{H}_{0} \mathrm{e}^{\mathrm{i} \mathbf{k} \cdot \mathbf{r}},
\end{aligned}
$$

where $\mathbf{k}=\left(k_{x}, k_{y}, k_{z}\right)^{\mathrm{T}}$ is the wave vector. Due to the continuity of the tangential component, $k_{\rho}=\sqrt{k_{x}^{2}+k_{y}^{2}}$ is identical in all layers and it suffices to derive the reflections and transmissions for all $k_{z}$-values using the continuity conditions at the material interfaces.

The reader should be aware of the different ways of numbering the layers. Here, we count the layers from bottom to top as sketched in Fig. 2. This is opposite to the numbering used in [11].

Fig. 2 Planar structure of a

\begin{tabular}{|c|c|}
\hline \multicolumn{2}{|c|}{ Layer $N$} \\
\hline \multicolumn{2}{|l|}{$\vdots$} \\
\hline Layer $i$ & $4^{Z}$ \\
\hline$\vdots$ & \\
\hline Layer 2 & Source \\
\hline
\end{tabular}
layered medium.

\subsubsection{Reflection Coefficients}

Starting with the simple case of two half-spaces, reflections and transmissions are occurring at a single layer interface. This directly implies that no multiple reflections need to be considered leading to the Fresnel reflection coefficients $R$. When splitting the field into transverse electric (TE) and transverse magnetic (TM) modes, one obtains for the two half-spaces $i$ and $j$ 


$$
\begin{aligned}
R_{i, j}^{\mathrm{TE}} & =\frac{\mu_{j} k_{z, i}-\mu_{i} k_{z, j}}{\mu_{j} k_{z, i}+\mu_{i} k_{z, j}}, \\
R_{i, j}^{\mathrm{TM}} & =\frac{\varepsilon_{j} k_{z, i}-\varepsilon_{i} k_{z, j}}{\varepsilon_{j} k_{z, i}+\varepsilon_{i} k_{z, j}},
\end{aligned}
$$

where $\varepsilon, \mu$ and $k_{z}$ are the permittivity, permeability and the normal component of the wave vector, respectively. For better readability the superscripts TE and TM will be omitted, if the equation holds for both cases. From the definitions (3), the antisymmetric property $R_{j, i}=-R_{i, j}$ of Fresnel reflection coefficients can be easily extracted.

Once we introduce finite layers, multiple reflections start occurring and the reflection coefficients need to be generalized. For the interface between layers $i$ and $j$ we then use

$$
\tilde{R}_{i, j}=\frac{R_{i, j}+\tilde{R}_{j, k} \mathrm{e}^{2 \mathrm{i} k_{z, j} d_{j}}}{1+R_{i, j} \tilde{R}_{j, k} \mathrm{e}^{2 \mathrm{i} k_{z, j} d_{j}}},
$$

where $d_{j}$ is the thickness of layer $j$ and the index $k$ represents the other layer next to layer $j$ (opposite to layer $i$ ). In Eq. (4), the two uppermost and the two lowermost layers are special cases. The topmost layer $N$ is a half-space and therefore has no reflections from above, so $\tilde{R}_{N, N+1}=0$. The second uppermost layer $N-1$ has no transmitted waves from top layer reflections (i.e. $\tilde{R}_{N, N+1} \mathrm{e}^{2 \mathrm{i} k_{z, N} d_{N}}=0$ although $d_{N} \rightarrow$ $\infty)$. This implies that the generalized reflection coefficient is equal to the Fresnel coefficient, $\tilde{R}_{N-1, N}=R_{N-1, N}$. For all other layers, the relation (4) can be applied from top to bottom to get all reflection coefficients of the form $\tilde{R}_{i, i+1}$. An equivalent procedure from bottom to top holds for the coefficients of the form $\tilde{R}_{i, i-1}$ where the two lowermost layers are special cases.

Equation (4) only holds in the case of a sourceless layer. Dipole sources in layered media are covered in Sect. 3.2, but nonetheless an additional term for the source layer $s$ has to be defined first. It reads

$$
\tilde{M}_{s}=\left[1-\tilde{R}_{s, s+1} \tilde{R}_{s, s-1} \mathrm{e}^{2 \mathrm{i} k_{z, s} d_{s}}\right]^{-1}
$$

and describes the magnitude enhancement or attenuation by reflection and transmission. There is no common name for this term, but in this work we will call it generalized source magnitude factor.

\subsubsection{Continuity at Layer Interfaces}

This last part about layered structures covers the propagation of waves through layers (cf. [11]). Again, they should be separated in TE- and TM-waves, but most equations are valid for both modes. For a complete coverage, four different cases must be considered due to upward $(\uparrow)$ and downward $(\downarrow)$ propagation and positive $(+)$ and negative $(-)$ superposition with reflected waves. First, we discuss a positive superposition and start with upward propagation. The wave in layer $i$ is then given 
by

$$
A_{i, \uparrow}^{+}\left[\mathrm{e}^{\mathrm{i} k_{z, i} z}+\tilde{R}_{i, i+1} \mathrm{e}^{-\mathrm{i} k_{z, i} z} \mathrm{e}^{\mathrm{i} k_{z, i} 2 z_{i, i+1}}\right],
$$

where $A_{i, \uparrow}^{+}$is the unknown amplitude and $z_{i, i+1}$ is the z-position of the boundary between layers $i$ and $i+1$. Assuming we know the amplitude $A_{i-1, \uparrow}^{+}$, we can enforce equality at the layer boundary $z_{i-1, i}$ to determine the amplitude in layer $i$. This results in the recursion

$$
A_{i, \uparrow}^{+}=A_{i-1, \uparrow}^{+} \frac{1+R_{i-1, i}}{1+R_{i-1, i} \tilde{R}_{i, i+1} \mathrm{e}^{\mathrm{i} k_{z, i} i d_{i}}} \mathrm{e}^{\mathrm{i}\left(k_{z, i-1}-k_{z, i}\right) z_{i-1, i}} .
$$

For downward propagating waves with field

$$
A_{i, \downarrow}^{+}\left[\mathrm{e}^{-\mathrm{i} k_{z, i} z}+\tilde{R}_{i, i-1} \mathrm{e}^{\mathrm{i} k_{z, i} z} \mathrm{e}^{-\mathrm{i} k_{z, i} 2 z_{i-1, i}}\right],
$$

the derivation is very similar and only the direction of propagation as well as the signs in the subscripts need to be flipped. After exploiting the antisymmetry of the Fresnel coefficients, the amplitude transfer factor reads

$$
A_{i, \downarrow}^{+}=A_{i+1, \downarrow}^{+} \frac{1-R_{i, i+1}}{1-R_{i, i+1} \tilde{R}_{i, i-1} \mathrm{e}^{\mathrm{i} k_{z, i} 2 d_{i}}} \mathrm{e}^{-\mathrm{i}\left(k_{z, i+1}-k_{z, i}\right) z_{i, i+1}} .
$$

The same can also be done for waves with negative reflection superposition. The fields now have the form

$$
A_{i, \uparrow}^{-}\left[\mathrm{e}^{\mathrm{i} k_{z, i} z}-\tilde{R}_{i, i+1} \mathrm{e}^{-\mathrm{i} k_{z, i} z} \mathrm{e}^{\mathrm{i} k_{z, i} 2 z_{i, i+1}}\right]
$$

for upward propagation and

$$
A_{i, \downarrow}^{-}\left[\mathrm{e}^{-\mathrm{i} k_{z, i} z}-\tilde{R}_{i, i-1} \mathrm{e}^{\mathrm{i} k_{z, i} z} \mathrm{e}^{-\mathrm{i} k_{z, i} 2 z_{i-1, i}}\right]
$$

for downward propagation. Assuming that the neighboring amplitude is known, the current one can be computed in a similar manner as above. Finally, this leads to the amplitude transfer factors

$$
\begin{aligned}
& A_{i, \uparrow}^{-}=A_{i-1, \uparrow}^{-} \frac{1-R_{i-1, i}}{1+R_{i-1, i} \tilde{R}_{i, i+1} \mathrm{e}^{\mathrm{i} k_{z, i} 2 d_{i}}} \mathrm{e}^{\mathrm{i}\left(k_{z, i-1}-k_{z, i}\right) z_{i-1, i}}, \\
& A_{i, \downarrow}^{-}=A_{i+1, \downarrow}^{-} \frac{1+R_{i, i+1}}{1-R_{i, i+1} \tilde{R}_{i, i-1} \mathrm{e}^{\mathrm{i} k_{z, i} 2 d_{i}}} \mathrm{e}^{-\mathrm{i}\left(k_{z, i+1}-k_{z, i}\right) z_{i, i+1}},
\end{aligned}
$$

for upward and downward propagation, respectively.

Similarly to Eq. (5), starting equations in the source layer can be defined. They are then used for the above iterative amplitude computation for all other layers. For TE-waves and a horizontally $(h)$ oriented source at distance $h$ from the lower layer boundary, they read as: 


$$
\begin{aligned}
& A_{s, \uparrow, h}^{\mathrm{TE}}=\left(1+\tilde{R}_{s, s-1}^{\mathrm{TE}} \mathrm{e}^{\mathrm{i} k_{z, s} 2 h}\right) \tilde{M}_{s}^{\mathrm{TE}}, \\
& A_{s, \downarrow, h}^{\mathrm{TE}}=\left(1+\tilde{R}_{s, s+1}^{\mathrm{TE}} \mathrm{e}^{\mathrm{i} k_{z, s} 2\left(d_{s}-h\right)}\right) \tilde{M}_{s}^{\mathrm{TE}},
\end{aligned}
$$

whereas for TM-waves, they are given by

$$
\begin{aligned}
& A_{s, \uparrow, h}^{\mathrm{TM}}=\left(-1+\tilde{R}_{s, s-1}^{\mathrm{TM}} \mathrm{e}^{\mathrm{i} k_{z, s} 2 h}\right) \tilde{M}_{s}^{\mathrm{TM}}, \\
& A_{s, \downarrow, h}^{\mathrm{TM}}=\left(-1+\tilde{R}_{s, s+1}^{\mathrm{TM}} \mathrm{e}^{\mathrm{i} k_{z, s} 2\left(d_{s}-h\right)}\right) \tilde{M}_{s}^{\mathrm{TM}} .
\end{aligned}
$$

In the case of a vertically ( $v$ ) oriented source, only TM-waves need to be considered and the starting amplitudes can be written as

$$
\begin{aligned}
& A_{s, \uparrow, v}^{\mathrm{TM}}=\left(1+\tilde{R}_{s, s-1}^{\mathrm{TM}} \mathrm{e}^{\mathrm{i} k_{z, s} 2 h}\right) \tilde{M}_{s}^{\mathrm{TM}} \\
& A_{s, \downarrow, v}^{\mathrm{TM}}=\left(1+\tilde{R}_{s, s+1}^{\mathrm{TM}} \mathrm{e}^{\mathrm{i} k_{z, s} 2\left(d_{s}-h\right)}\right) \tilde{M}_{s}^{\mathrm{TM}} .
\end{aligned}
$$

\subsection{Layered Dipole}

With the reflection coefficients at hand, we can now proceed to compute the emission of a dipole in a stratified medium. As before, we refrain from giving a full derivation and only present the main steps. To obtain the physical fields, all propagating $\left(k_{z}^{2}>0\right)$ and evanescent $\left(k_{z}^{2}<0\right)$ contributions have to be properly summed up. One therefore has to evaluate Sommerfeld integrals of the form $[11,12,13]$

$$
F_{i j}=\frac{1}{4 \pi} \int_{0}^{\infty} G_{i j}^{F} J_{0}\left(k_{\rho} \rho\right) k_{\rho} \mathrm{d} k_{\rho} .
$$

Here, $J_{v}(\cdot)$ is the Bessel function of first kind of order $v$ and $G_{i j}^{F}$ is the Green's function operator for a field component $F$ in coordinate direction $i$ of a dipole with axis in direction $j$. An additional factor $1 / 2$ was already pulled out for a simplification of the Green's functions later in this section. The integrand contains singularities along the real axis, so one typically moves the integration path into the complex plane. In our case, we employ a sinusoidal path as sketched in Fig. 3 , where $k_{L \text {,max }}$ and $k_{L \text {,min }}$ denote the largest and smallest wavenumber in the layer materials, respectively. As an additional complication, the integration along the tail can be highly oscillatory. This is why the integration is often converging slowly when the observation point is close to the source. There are several methods proposed in literature (e.g. see [15]) to accelerate convergence in such cases. Here, for the numerical integration, we simply subtract the direct term, which can be computed analytically. The analytic result is then added afterwards. 


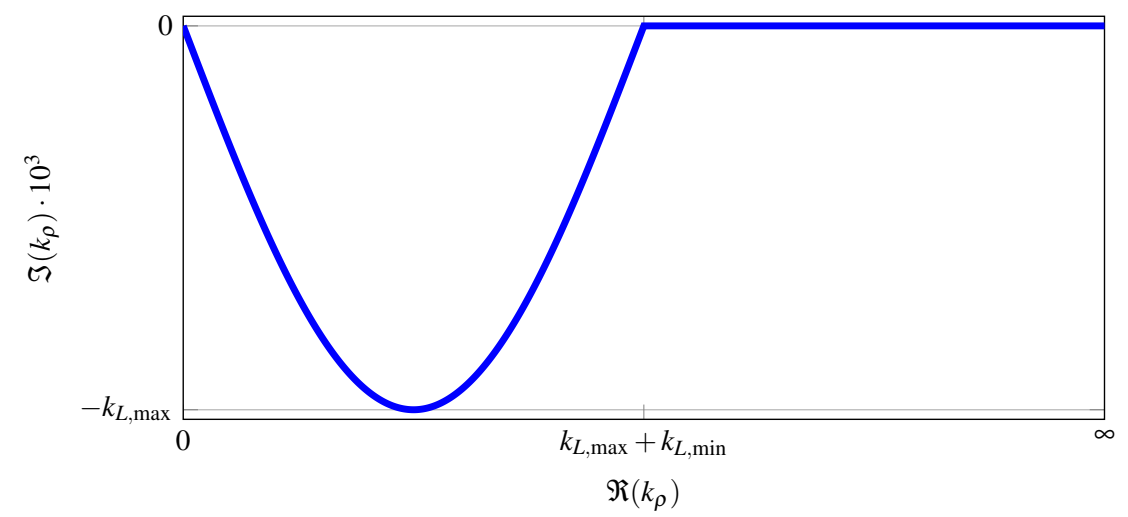

Fig. 3 Schematic of the Sommerfeld integration path to avoid singularities on the real axis.

The fields $\mathbf{F}=\left(E_{x}, E_{y}, E_{z}, H_{x}, H_{y}, H_{z}\right)^{\mathrm{T}}$ of the layered dipole expansion $e$ can be written as

$$
\mathbf{F}=\mathrm{A}_{e, r} \mathbf{x}_{e},
$$

where $\mathbf{x}_{e}=\left(x_{e, x}, x_{e, y}, x_{e, z}\right)^{\mathrm{T}}$ is the expansion strength and $\mathrm{A}_{e, r}$ is the layered dipole matrix for observation location $r$ with radial distance $\rho$. The full layered dipole matrix reads

$$
\mathrm{A}_{e, r}=\frac{\mathrm{W}_{b c}}{4 \pi} \int_{0}^{\infty}\left[\begin{array}{lll}
G_{x x}^{E} & G_{x y}^{E} & G_{x z}^{E} \\
G_{y x}^{E} & G_{y y}^{E} & G_{y z}^{E} \\
G_{z x}^{E} & G_{z y}^{E} & G_{z z}^{E} \\
G_{x x}^{H} & G_{x y}^{H} & G_{x z}^{H} \\
G_{y x}^{H} & G_{y y}^{H} & G_{y z}^{H} \\
G_{z x}^{H} & G_{z y}^{H} & G_{z z}^{H}
\end{array}\right]_{e, r} J_{0}\left(k_{\rho} \rho\right) k_{\rho} \mathrm{d} k_{\rho},
$$

where the row-weights $\mathrm{W}_{b c}=\operatorname{diag}\left(1,1, \varepsilon_{s} / \varepsilon_{o}, 1,1, \mu_{s} / \mu_{o}\right)$ for source layer $s$ and observation layer $o$ were introduced to ensure that the continuity conditions are fulfilled at the material interfaces. The integration in (19) is meant to be computed element-wise and the Green's functions act as operators in space.

To compute the Green's functions in (19), we first derive them from Green's functions for the vector potential $\mathbf{A}$ and the scalar potential $\phi[16]$ as

$$
\begin{aligned}
& G_{x_{i} x_{j}}^{E}=\mathrm{i} \omega G_{x_{i} x_{j}}^{A}-\frac{1}{\mathrm{i} \omega} \frac{\partial^{2}}{\partial x_{i} \partial x_{j}} G_{x_{j}}^{\phi}, \\
& G_{x_{i} x_{j}}^{H}=\frac{1}{\mu}\left(\frac{\partial}{\partial x_{i_{+}}} G_{x_{i_{-}} x_{j}}^{A}-\frac{\partial}{\partial x_{i_{-}}} G_{x_{i_{+}} x_{j}}^{A}\right) .
\end{aligned}
$$

Here, $G_{x_{i} x_{j}}^{H}$ results from a curl operation, so $x_{i_{-}}$and $x_{i_{+}}$represent the previous and next coordinate direction relative to $x_{i}$, respectively. Since the layers are stacked in vertical direction, the Green's functions are only dependent on $z$, while the horizon- 
tal dependence comes from the Bessel function $J_{0}\left(k_{\rho} \rho\right)$. Therefore, all derivatives with respect to $x$ and $y$ do not affect the Green's functions. Furthermore, some of the vector potential Green's functions are zero, namely $G_{x y}^{A}=G_{y x}^{A}=G_{x z}^{A}=G_{y z}^{A}=0$. Others are identical due to symmetry, such as $G_{x x}^{A}=G_{y y}^{A}, G_{x}^{\phi}=G_{y}^{\phi}$. To derive the final expressions, three cases must be distinguished:

- observation in source layer,

- observation above source layer,

- observation below source layer.

\subsubsection{Observation in Source Layer}

For the case when the source and the observation point lie in the same layer, only the indirect terms originating from reflections are integrated. To do so, we first specify the amplitude coefficients [13] for horizontal dipoles

$$
\begin{aligned}
& A_{h}=\mathrm{e}^{\mathrm{i} k_{z, s}\left(d_{s}-h\right)} \tilde{R}_{s, s+1}^{\mathrm{TE}}\left[\mathrm{e}^{\mathrm{i} k_{z, s}\left(d_{s}-h\right)}+\tilde{R}_{s, s-1}^{\mathrm{TE}} \mathrm{e}^{\mathrm{i} k_{z, s}\left(d_{s}+h\right)}\right] \tilde{M}_{s}^{\mathrm{TE}}, \\
& B_{h}=\mathrm{e}^{\mathrm{i} k_{z, s}\left(d_{s}-h\right)} \tilde{R}_{s, s+1}^{\mathrm{TM}}\left[\mathrm{e}^{\mathrm{i} k_{z, s}\left(d_{s}-h\right)}-\tilde{R}_{s, s-1}^{\mathrm{TM}} \mathrm{e}^{\mathrm{i} k_{z, s}\left(d_{s}+h\right)}\right] \tilde{M}_{s}^{\mathrm{TM}}, \\
& C_{h}=\mathrm{e}^{\mathrm{i} k_{z, s} h} \tilde{R}_{s, s-1}^{\mathrm{TE}}\left[\mathrm{e}^{\mathrm{i} k_{z, s} h}+\tilde{R}_{s, s+1}^{\mathrm{TE}} \mathrm{e}^{\mathrm{i} k_{z, s}\left(2 d_{s}-h\right)}\right] \tilde{M}_{s}^{\mathrm{TE}}, \\
& D_{h}=\mathrm{e}^{\mathrm{i} k_{z, s} h} \tilde{R}_{s, s-1}^{\mathrm{TM}}\left[\mathrm{e}^{\mathrm{i} k_{z, s} h}-\tilde{R}_{s, s+1}^{\mathrm{TM}} \mathrm{e}^{\mathrm{i} k_{z, s}\left(2 d_{s}-h\right)}\right] \tilde{M}_{s}^{\mathrm{TM}} .
\end{aligned}
$$

Similarly, for vertical dipoles

$$
\begin{aligned}
& A_{v}=\mathrm{e}^{\mathrm{i} k_{z, s} h} \tilde{R}_{s, s-1}^{\mathrm{TM}}\left[\mathrm{e}^{\mathrm{i} k_{z, s} h}+\tilde{R}_{s, s+1}^{\mathrm{TM}} \mathrm{e}^{\mathrm{i} k_{z, s}\left(2 d_{s}-h\right)}\right] \tilde{M}_{s}^{\mathrm{TM}}, \\
& B_{v}=\mathrm{e}^{\mathrm{i} k_{z, s}\left(d_{s}-h\right)} \tilde{R}_{s, s+1}^{\mathrm{TM}}\left[\mathrm{e}^{\mathrm{i} k_{z, s}\left(d_{s}-h\right)}+\tilde{R}_{s, s-1}^{\mathrm{TM}} \mathrm{e}^{\mathrm{i} k_{z, s}\left(d_{s}+h\right)}\right] \tilde{M}_{s}^{\mathrm{TM}},
\end{aligned}
$$

where the naming conventions from Sect. 3.1.1 were adopted. With these coefficients at hand, we can explicitly state the Green's functions as 


$$
\begin{aligned}
& G_{x x}^{A}=G_{y y}^{A}=-\frac{\mu_{s}}{\mathrm{i} k_{z, s}}\left[A_{h} \mathrm{e}^{-\mathrm{i} k_{z, s} z}+C_{h} \mathrm{e}^{\mathrm{i} k_{z, s} z}\right], \\
& G_{z z}^{A}=-\frac{\mu_{s}}{\mathrm{i} k_{z, s}}\left[A_{\nu} \mathrm{e}^{\mathrm{i} k_{z, s} z}+B_{v} \mathrm{e}^{-\mathrm{i} k_{z, s} z}\right], \\
& G_{z x}^{A}=-\frac{\mu_{s}}{k_{\rho}^{2}}\left[\left(A_{h}+B_{h}\right) \mathrm{e}^{-\mathrm{i} k_{z, s} z}-\left(C_{h}+D_{h}\right) \mathrm{e}^{\mathrm{i} k_{z, s} z}\right] \frac{\partial}{\partial x}, \\
& G_{z y}^{A}=-\frac{\mu_{s}}{k_{\rho}^{2}}\left[\left(A_{h}+B_{h}\right) \mathrm{e}^{-\mathrm{i} k_{z, s} z}-\left(C_{h}+D_{h}\right) \mathrm{e}^{\mathrm{i} k_{z, s} z}\right] \frac{\partial}{\partial y}, \\
& G_{x}^{\phi}=G_{y}^{\phi}=-\frac{1}{\varepsilon_{s} \mathrm{i} k_{z, s}}\left[\frac{k_{s}^{2} A_{h}+k_{z, s}^{2} B_{h}}{k_{\rho}^{2}} \mathrm{e}^{-\mathrm{i} k_{z, s} z}+\frac{k_{s}^{2} C_{h}+k_{z, s}^{2} D_{h}}{k_{\rho}^{2}} \mathrm{e}^{\mathrm{i} k_{z, s} z}\right], \\
& G_{z}^{\phi}=-\frac{1}{\varepsilon_{s} \mathrm{i} k_{z, s}}\left[A_{\nu} \mathrm{e}^{\mathrm{i} k_{z, s} z}+B_{v} \mathrm{e}^{-\mathrm{i} k_{z, s} z}\right] .
\end{aligned}
$$

Inserting these expressions into (20), evaluating the derivatives and integrating the result yields the full indirect electromagnetic field matrix. Afterwards, the contributions of an identical dipole in a homogeneous medium needs to be added to obtain the final result.

\subsubsection{Observation above Source Layer}

When the observation point is in layer $o$ above the source, the amplitude transitions from Sect. 3.1.2 are required. To find the Green's functions in this case, multiple steps need to be performed. First, the Green's functions for the source layer (23) are evaluated at the upper layer boundary of the source layer. They can be reformulated such that their amplitudes have the form of the factors $A_{s, \uparrow}$ in (14a), (15a) or (16a), depending on orientation and wave type. The propagation through the layers can then be computed by the expressions given in Sect. 3.1.2. To simplify the computation, it is helpful to split the amplitude into a source term and a propagation factor. The missing propagation factor is retrieved by starting with a unit amplitude factor in the source layer and iteratively applying Eqs. (7) or (12) until the observation layer has been reached. With the amplitudes given, the Green's functions for the potentials can be stated as

$$
\begin{aligned}
G_{x x}^{A}=G_{y y}^{A}=-\frac{\mu_{s}}{\mathrm{i} k_{z, s}}\left[A_{s, \uparrow, h}^{\mathrm{TE}} A_{o, \uparrow}^{+, \mathrm{TE}}\left(\mathrm{e}^{\mathrm{i} k_{z, o} z}+\tilde{R}_{o, o+1}^{\mathrm{TE}} \mathrm{e}^{\mathrm{i} k_{z, o}\left(2 z_{o, o+1}-z\right)}\right)\right], \\
G_{z z}^{A}=-\frac{\mu_{s}}{\mathrm{i} k_{z, s}}\left[A_{s, \uparrow, v}^{\mathrm{TM}} A_{o, \uparrow}^{+, \mathrm{TM}}\left(\mathrm{e}^{\mathrm{i} k_{z, o} z}+\tilde{R}_{o, o+1}^{\mathrm{TM}} \mathrm{e}^{\mathrm{i} k_{z, o}\left(2 z_{o, o+1}-z\right)}\right)\right], \\
G_{z x}^{A}=-\frac{\mu_{s}}{k_{\rho}^{2}}\left[-A_{s, \uparrow, h}^{\mathrm{TE}} A_{o, \uparrow}^{-, \mathrm{TE}}\left(\mathrm{e}^{\mathrm{i} k_{z, o} z}-\tilde{R}_{o, o+1}^{\mathrm{TE}} \mathrm{e}^{\mathrm{i} k_{z, o}\left(2 z_{o, o+1}-z\right)}\right)-\right. \\
\left.-A_{s, \uparrow, h}^{\mathrm{TM}} A_{o, \uparrow}^{+, \mathrm{TM}}\left(\mathrm{e}^{\mathrm{i} k_{z, o} z}+\tilde{R}_{o, o+1}^{\mathrm{TM}} \mathrm{e}^{\mathrm{i} k_{z, o}\left(2 z_{o, o+1}-z\right)}\right)\right] \frac{\partial}{\partial x},
\end{aligned}
$$




$$
\begin{aligned}
& G_{z y}^{A}=-\frac{\mu_{s}}{k_{\rho}^{2}}\left[-A_{s, \uparrow, h}^{\mathrm{TE}} A_{o, \uparrow}^{-, \mathrm{TE}}\left(\mathrm{e}^{\mathrm{i} k_{z, o} z}-\tilde{R}_{o, o+1}^{\mathrm{TE}} \mathrm{e}^{\mathrm{i} k_{z, o}\left(2 z_{o, o+1}-z\right)}\right)-\right. \\
& \left.-A_{s, \uparrow, h}^{\mathrm{TM}} A_{o, \uparrow}^{+, \mathrm{TM}}\left(\mathrm{e}^{\mathrm{i} k_{z, o} z}+\tilde{R}_{o, o+1}^{\mathrm{TM}} \mathrm{e}^{\mathrm{i} k_{z, o}\left(2 z_{o, o+1}-z\right)}\right)\right] \frac{\partial}{\partial y}, \\
& G_{x}^{\phi}=G_{y}^{\phi}=-\frac{1}{\varepsilon_{s} \mathrm{i} k_{z, s}}\left[\frac{k_{s}^{2}}{k_{\rho}^{2}} A_{s, \uparrow, h}^{\mathrm{TE}} A_{o, \uparrow}^{+, \mathrm{TE}}\left(\mathrm{e}^{\mathrm{i} k_{z, o} z}+\tilde{R}_{o, o+1}^{\mathrm{TE}} \mathrm{e}^{\mathrm{i} k_{z, o}\left(2 z_{o, o+1}-z\right)}\right)+\right. \\
& \left.+\frac{k_{z, s}^{2}}{k_{\rho}^{2}} A_{s, \uparrow, h}^{\mathrm{TM}} A_{o, \uparrow}^{-, \mathrm{TM}}\left(\mathrm{e}^{\mathrm{i} k_{z, o} z}-\tilde{R}_{o, o+1}^{\mathrm{TM}} \mathrm{e}^{\mathrm{i} k_{z, o}\left(2 z_{o, o+1}-z\right)}\right)\right], \\
& G_{z}^{\phi}=-\frac{1}{\varepsilon_{s} \mathrm{i} k_{z, s}}\left[A_{s, \uparrow, v}^{\mathrm{TM}} A_{o, \uparrow}^{+, \mathrm{TM}}\left(\mathrm{e}^{\mathrm{i} k_{z, o} z}+\tilde{R}_{o, o+1}^{\mathrm{TM}} \mathrm{e}^{\mathrm{i} k_{z, o}\left(2 z_{o, o+1}-z\right)}\right)\right] .
\end{aligned}
$$

From here, the same steps as discussed in the previous section must be carried out to obtain the final matrix. In this case, no direct term needs to be added.

\subsubsection{Observation below Source Layer}

The case when the observation layer $o$ is located below the source layer is very similar to the one above, but requires a change in the propagation direction. Hence, the final formulas read

$$
\begin{aligned}
& G_{x x}^{A}=G_{y y}^{A}=-\frac{\mu_{s}}{\mathrm{i} k_{z, s}}\left[A_{s, \downarrow}^{\mathrm{TE}} A_{o, \downarrow}^{+, \mathrm{TE}}\left(\mathrm{e}^{-\mathrm{i} k_{z, o} z}+\tilde{R}_{o, o-1}^{\mathrm{TE}} \mathrm{e}^{-\mathrm{i} k_{z, o}\left(2 z_{o-1, o}-z\right)}\right)\right], \\
& G_{z z}^{A}=-\frac{\mu_{s}}{\mathrm{i} k_{z, s}}\left[A_{s, \downarrow, v}^{\mathrm{TM}} A_{o, \downarrow}^{+, \mathrm{TM}}\left(\mathrm{e}^{-\mathrm{i} k_{z, o} z}+\tilde{R}_{o, o-1}^{\mathrm{TM}} \mathrm{e}^{-\mathrm{i} k_{z, o}\left(2 z_{o-1, o}-z\right)}\right)\right], \\
& G_{z x}^{A}=-\frac{\mu_{s}}{k_{\rho}^{2}}\left[A_{s, \downarrow, h}^{\mathrm{TE}} A_{o, \downarrow}^{-, \mathrm{TE}}\left(\mathrm{e}^{-\mathrm{i} k_{z, o} z}-\tilde{R}_{o, o-1}^{\mathrm{TE}} \mathrm{e}^{-\mathrm{i} k_{z, o}\left(2 z_{o-1, o}-z\right)}\right)+\right. \\
& \left.+A_{s, \downarrow, h}^{\mathrm{TM}} A_{o, \downarrow}^{+, \mathrm{TM}}\left(\mathrm{e}^{-\mathrm{i} k_{z, o} z}+\tilde{R}_{o, o-1}^{\mathrm{TM}} \mathrm{e}^{-\mathrm{i} k_{z, o}\left(2 z_{o-1, o}-z\right)}\right)\right] \frac{\partial}{\partial x}, \\
& G_{z y}^{A}=-\frac{\mu_{s}}{k_{\rho}^{2}}\left[A_{s, \downarrow, h}^{\mathrm{TE}} A_{o, \downarrow}^{-, \mathrm{TE}}\left(\mathrm{e}^{-\mathrm{i} k_{z, o} z}-\tilde{R}_{o, o-1}^{\mathrm{TE}} \mathrm{e}^{-\mathrm{i} k_{z, o}\left(2 z_{o-1, o}-z\right)}\right)+\right. \\
& \left.+A_{s, \downarrow, h}^{\mathrm{TM}} A_{o, \downarrow}^{+, \mathrm{TM}}\left(\mathrm{e}^{-\mathrm{i} k_{z, o} z}+\tilde{R}_{o, o-1}^{\mathrm{TM}} \mathrm{e}^{-\mathrm{i} k_{z, o}\left(2 z_{o-1, o}-z\right)}\right)\right] \frac{\partial}{\partial y}, \\
& G_{x}^{\phi}=G_{y}^{\phi}=-\frac{1}{\varepsilon_{s} \mathrm{i} k_{z, s}}\left[\frac{k_{s}^{2}}{k_{\rho}^{2}} A_{s, \downarrow, h}^{\mathrm{TE}} A_{o, \downarrow}^{+, \mathrm{TE}}\left(\mathrm{e}^{-\mathrm{i} k_{z, o} z}+\tilde{R}_{o, o-1}^{\mathrm{TE}} \mathrm{e}^{-\mathrm{i} k_{z, o}\left(2 z_{o-1, o}-z\right)}\right)+\right. \\
& \left.+\frac{k_{z, s}^{2}}{k_{\rho}^{2}} A_{s, \downarrow, h}^{\mathrm{TM}} A_{o, \downarrow}^{-, \mathrm{TM}}\left(\mathrm{e}^{-\mathrm{i} k_{z, o} z}-\tilde{R}_{o, o-1}^{\mathrm{TM}} \mathrm{e}^{-\mathrm{i} k_{z, o}\left(2 z_{o-1, o}-z\right)}\right)\right], \\
& G_{z}^{\phi}=-\frac{1}{\varepsilon_{s} \mathrm{i} k_{z, s}}\left[A_{s, \downarrow, v}^{\mathrm{TM}} A_{o, \downarrow}^{+, \mathrm{TM}}\left(\mathrm{e}^{-\mathrm{i} k_{z, o} z}+\tilde{R}_{o, o-1}^{\mathrm{TM}} \mathrm{e}^{-\mathrm{i} k_{z, o}\left(2 z_{o-1, o}-z\right)}\right)\right] .
\end{aligned}
$$


Again, the final matrix is found by transformation and integration and no direct term needs to be added.

\section{Electron Energy Loss Spectroscopy}

As discussed in the introduction, electron energy loss spectroscopy (EELS) is a technique to experimentally determine optical excitation spectra using a scanning transmission electron microscope (STEM). To simulate the physical processes in an EELS measurement requires a few extensions to MMP which will be discussed in the following.

\subsection{Electron Beam Expansion}

First, the electromagnetic fields caused by a relativistic electron beam must be computed. Here, the only free parameters are the position and the velocity $v$ of the electrons. Assuming a propagation along the $z$-axis, the electric and magnetic field can be written as in [2]

$$
\begin{aligned}
& E_{x}=-\frac{2 e \omega}{v^{2} \gamma \varepsilon} \mathrm{e}^{\mathrm{i} \omega \frac{z}{v}} K_{1}\left(\frac{\omega \rho}{v \gamma}\right) \frac{x}{\rho}, \\
& E_{y}=-\frac{2 e \omega}{v^{2} \gamma \varepsilon} \mathrm{e}^{\mathrm{i} \omega \frac{z}{v}} K_{1}\left(\frac{\omega \rho}{v \gamma}\right) \frac{y}{\rho}, \\
& E_{z}=\frac{2 e \omega}{v^{2} \gamma \varepsilon} \mathrm{e}^{\mathrm{i} \omega \frac{z}{v}} K_{0}\left(\frac{\omega \rho}{v \gamma}\right) \frac{\mathrm{i}}{\gamma}, \\
& H_{x}=\frac{2 e \omega}{v \gamma} \mathrm{e}^{\mathrm{i} \omega \frac{z}{v}} K_{1}\left(\frac{\omega \rho}{v \gamma}\right) \frac{y}{\rho}, \\
& H_{y}=-\frac{2 e \omega}{v \gamma} \mathrm{e}^{\mathrm{i} \omega \frac{z}{v}} K_{1}\left(\frac{\omega \rho}{v \gamma}\right) \frac{x}{\rho}, \\
& H_{z}=0 .
\end{aligned}
$$

Here, we introduced

- the elementary charge $e$,

- the Lorentz contraction factor $\gamma=\left(\sqrt{1-v^{2} / c^{2}}\right)^{-1}$,

- the speed of light in dielectric material $c$,

- the modified Bessel function of second kind $K_{V}(\cdot)$ of order $v$,

- the distance to the beam $\rho=\sqrt{\left(x-x_{0}\right)^{2}+\left(y-y_{0}\right)^{2}}$.

The electric and magnetic field caused by an electron beam are decaying exponentially from the center and have a mainly radial and azimuthal orientation, respec- 
tively. They are therefore similar to the fields generated by a line current but include a retardation effect.

\subsection{Electron Energy Loss Computation}

With the source terms at hand, we use MMP to compute the scattering caused by a structure close to the electron beam. To then obtain an actual loss spectrum, some further post-processing is required. The frequency-dependent total energy loss $P(\omega)$ can be computed by integration of the energy loss along the electron path [2] as

$$
P(\omega)=\frac{1}{\pi \hbar \omega} \int_{-\infty}^{\infty} \Re\left\{\mathrm{e}^{-\mathrm{i} \omega t} v E_{z}^{\text {scat }}(z(t), \omega)\right\} \mathrm{d} t,
$$

where the electron beam direction is again chosen to be in local z-direction without loss of generality. By inserting the transformation $t=\frac{z(t)-z_{0}}{v}$ and using setting $z_{0}=$ 0 , the integration can be performed over space instead of time. Thus, we need to evaluate

$$
P(\omega)=\frac{1}{\pi \hbar \omega} \int_{-\infty}^{\infty} \Re\left\{\mathrm{e}^{-\mathrm{i} \omega \frac{z}{v}} E_{z}^{\text {scat }}(z, \omega)\right\} \mathrm{d} z,
$$

which can be done using an appropriate numerical quadrature rule.

\section{Numerical Experiments}

In the following, we present a series of numerical experiments to demonstrate the convergence behavior and performance of our MMP implementation. For the numerical quadrature, we implemented a routine similar to the one proposed in [17]. For the evaluation of Bessel functions with complex arguments, the Fortran library of D.E. Amos [18] was ported to C++, giving highly accurate values at the cost of somewhat limited performance. As the main parts of MMP contain linear algebra, the versatile and powerful library Eigen [19] was used for all vector or matrix algebra functions.To accelerate the computation, a basic parallelization was implemented using Intels Math Kernel Library (MKL) [20] and Threading Building Blocks (TBB) [21] library. 


\subsection{Plane Wave Excitation of a Dielectric Sphere}

The first example serves solely to test the convergence of our mesh-based MMP approach. To this end, we model a dielectric sphere with relative permittivity $\varepsilon_{r}=4$. We excite it from the top by a plane wave with a wavelength equal to the diameter of the sphere. To compute the electromagnetic fields, we first need to fix the expansions and matching points. We start with the mesh and the corresponding distribution shown in Fig. 1.

Then, the excited fields can be computed following the standard MMP procedure. The real part of the scattered electric field on the polarization axis (in-plane) and the real part of the scattered magnetic field (out-of-plane) are depicted in Fig. 4 in a vertical cut plane through the center of the sphere.
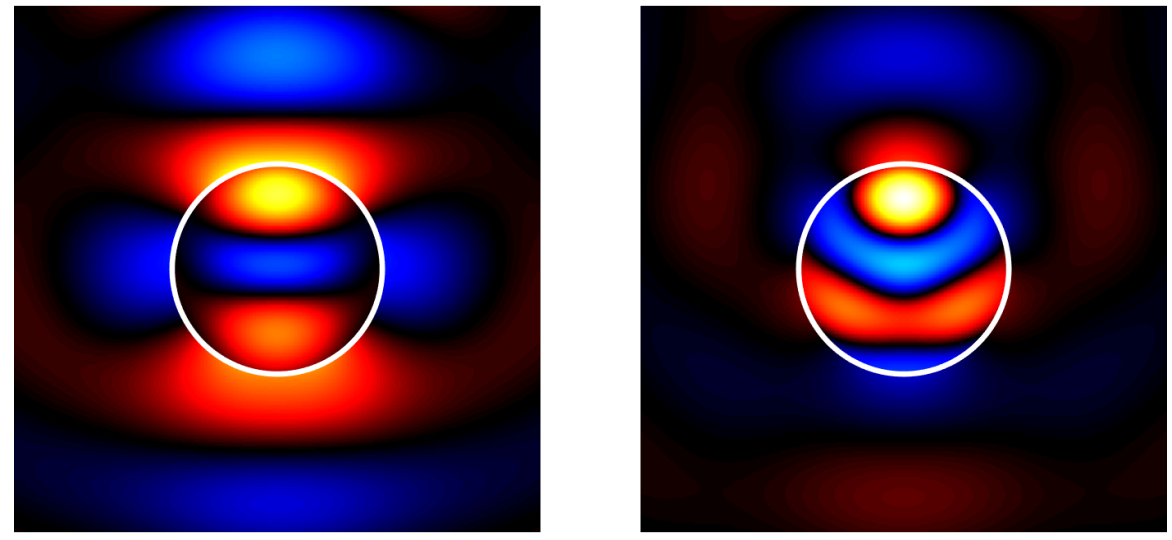

Fig. 4 Real part of the scattered electric and the scattered magnetic field of a dielectric sphere excited by a plane wave.

\subsection{Properties of Mesh-Based MMP}

To determine optimal parameters for an efficient and accurate numerical simulation with the mesh-based MMP approach, a series of numerical convergence studies was performed.

\subsubsection{Mesh-Refinement}

First, we test how many mesh elements are required to model the fields accurately. For this purpose, the mesh was successively refined. To compute error estimates, the field mismatch is integrated over the entire surface using a quadrature rule of higher 
order than the one used to generate the matching points. The graph in Fig. 5 shows the behavior of relative and absolute error over the number of mesh elements. We

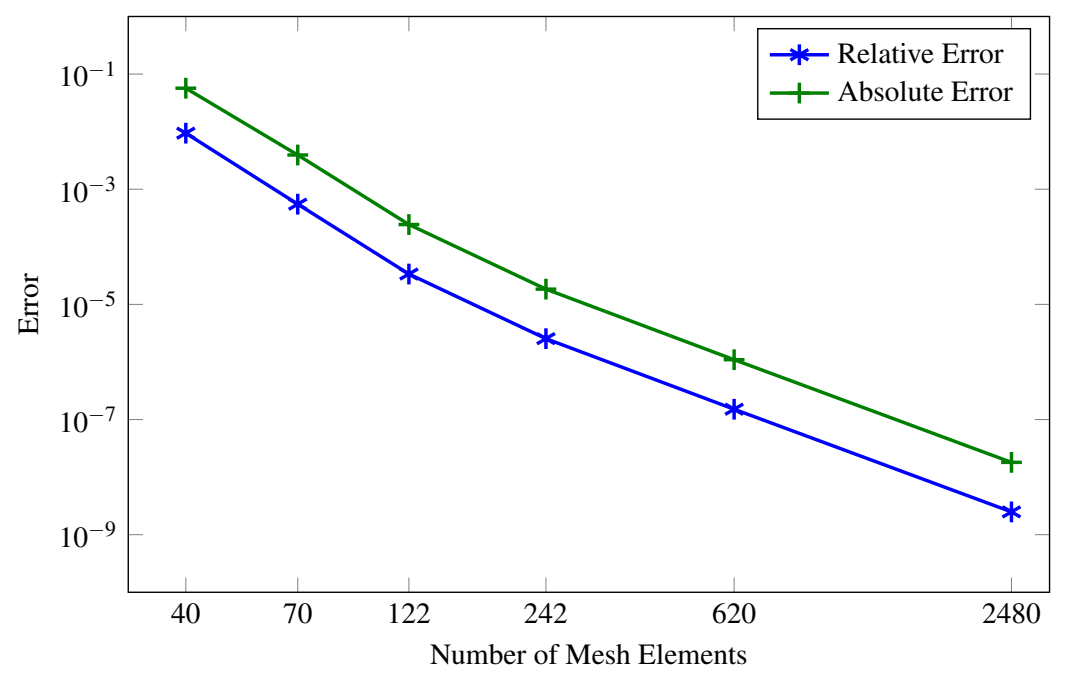

Fig. 5 Mesh refinement to observe convergence of the absolute and relative error using the example of a sphere excited by a plane wave.

observe an exponential decay with a slightly flattened slope after a kink at 122 elements. Since a relative error of around $10^{-5}$ is sufficient for most practical purposes, we will use the mesh with 122 elements for all further tests.

\subsubsection{Quadrature-Refinement}

The next important parameter is the order of the quadrature rule applied on the mesh elements for the matching point placement. The quadrature order directly influences the factor of over-determination of the MMP system. Naturally, this has a large impact on the memory consumption and on the computational time. Table 1 below shows the over-determination depending on the quadrature order.

Table 1 Over-determination caused by given quadrature order

\begin{tabular}{cc}
\hline Quadrature Order & Over-Determination \\
\hline 1 & 1 \\
2 & 3 \\
3 & 6 \\
4 & 6 \\
\hline
\end{tabular}


Since the rule of $1^{\text {st }}$ order only has one node in the center, it leads to a square MMP matrix. Unfortunately, it is well known that a square system can result in oscillatory or unphysical solutions. This behavior can also be observed in the error plot depicted in Fig. 6. On the other hand, triangular quadrature rules of $3^{\text {rd }}$ and

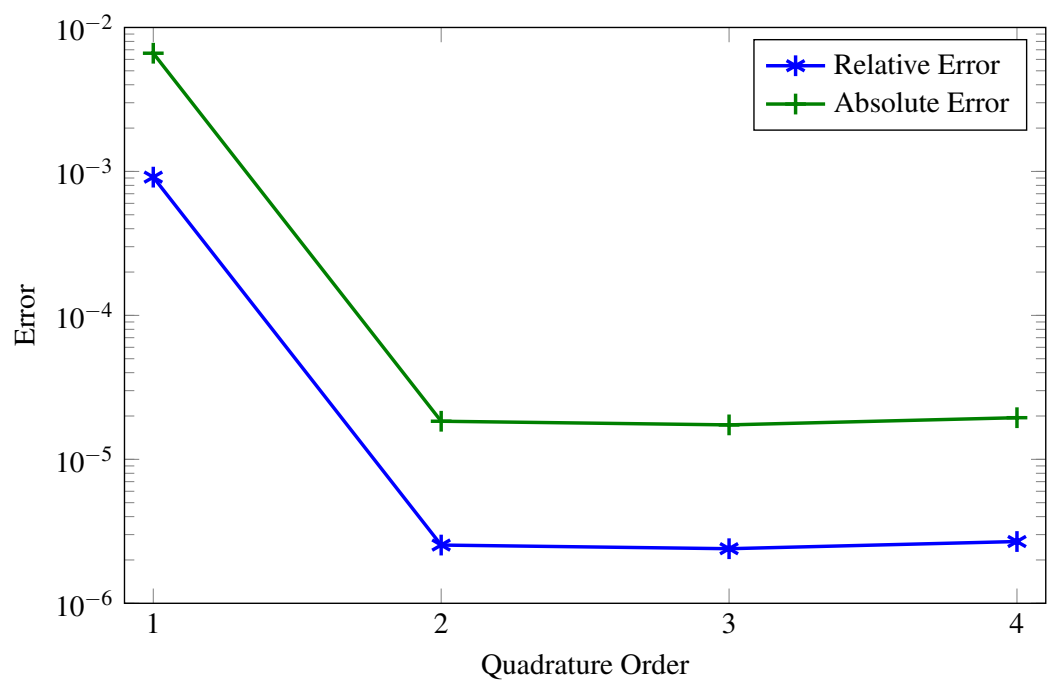

Fig. 6 Absolute and relative error for a sphere excited by a plane wave as a function of the quadrature order.

$4^{\text {th }}$ order have the same number of nodes, so we are left with the choice between a $2^{\text {nd }}$ order and a $4^{\text {th }}$ order rule. From the data in Fig. 6 we clearly see that a $4^{\text {th }}$ order rule does not provide any extra accuracy, so we employ $2^{\text {nd }}$ order rules for the distribution of matching points in all further MMP calculations.

\subsubsection{Mesh Element Order}

Finally, we briefly discuss the use of curvilinear elements vs. using straight elements. However, since the geometry only affects the preprocessing, namely the generation of expansions and matching points, it has no significant influence of the computational time. It therefore makes sense to use the most accurate geometry representation, i.e., curvilinear elements, if available. To support this statement, Table 2 shows the errors for both kind of elements for the spherical test system.

As expected, we find that the error reduces significantly when using curvilinear elements. 
Table 2 Absolute and relative errors for planar or curvilinear mesh elements using the example of a sphere excited by a plane wave.

\begin{tabular}{lcc}
\hline Error & Linear & Curved \\
\hline Relative & $2.0397 \cdot 10^{-5}$ & $2.5381 \cdot 10^{-6}$ \\
Absolute & $1.4712 \cdot 10^{-4}$ & $1.8393 \cdot 10^{-5}$ \\
\hline
\end{tabular}

\subsubsection{Expansion Distance to Particle Surface}

The final test of our MMP implementation concerns the influence of the expansion distance. For this particular case, we switch to a more challenging test geometry. Specifically, we will use a split-ring resonator similar to the one studied in [22]. The mesh is shown in Fig. 7 and the structure is excited by an electron beam at the position indicated by the red dot. Then, an electron energy loss spectrum is com-

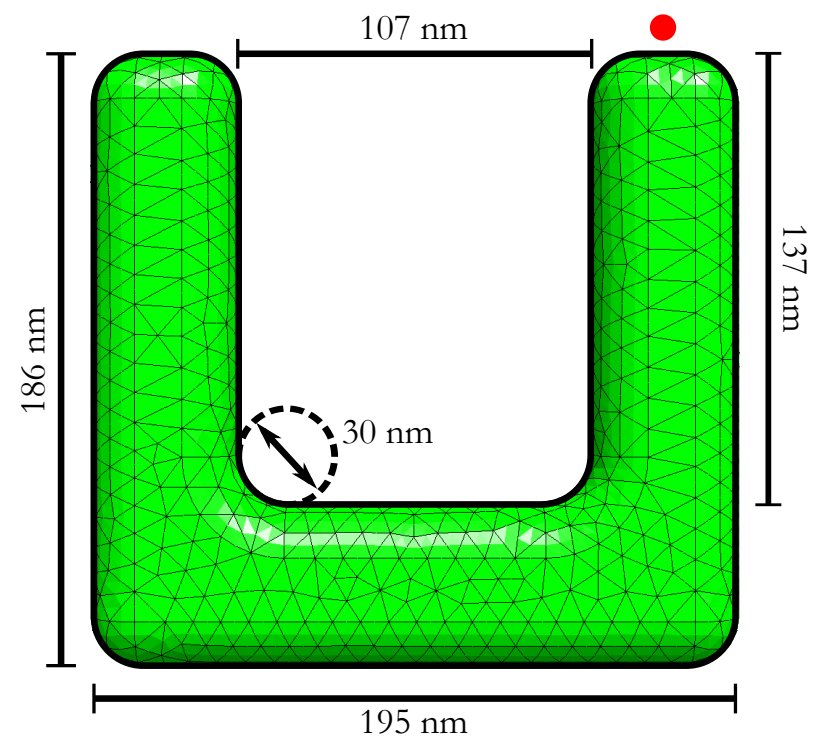

Fig. 7 Split-ring resonator geometry and mesh to show the experimental setup. The red dot indicates the location of the electron beam excitation.

puted for different distances of the expansions to the split-ring surface, starting at half the minimal curvature and going up to $90 \%$ thereof. The resulting spectra are depicted in Fig. 8. These results clearly demonstrate the importance and difficulty of placing the expansions optimally. For short distances, the signal is strongly suppressed. When moving the expansions further away, the spectrum converges towards its correct solution. For our example, the result does not change for distances above $d=13.5 \mathrm{~nm}$. However, when the expansions are placed even further away from the surface, their locations become too close to each other. As a result, the condition 


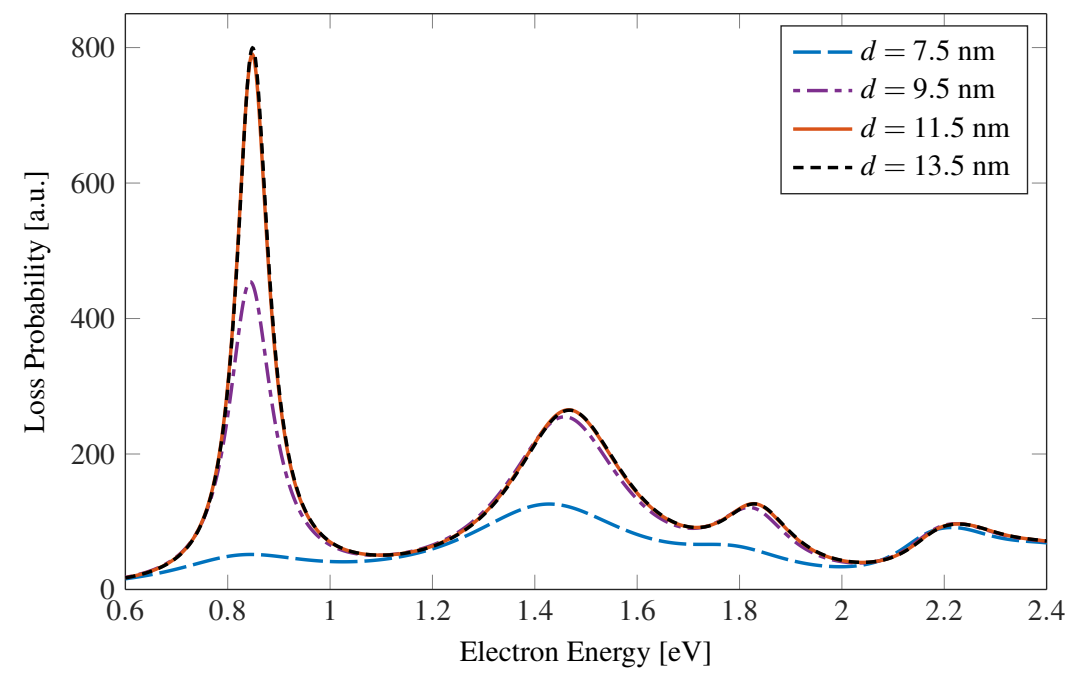

Fig. 8 Electron-energy loss spectra for different distances of the expansions to the surface. Increasing the distance improves the accuracy of the method considerably.

number of the MMP matrix deteriorates, which can lead to significant instabilities. We find that using a distance of around $90 \%$ of the smallest curvature radius in the geometry works well in most cases.

\subsection{Electron Energy Loss Spectroscopy of a Plasmonic Split-Ring Resonator in Free Space}

After the convergence studies in the previous sections, we now demonstrate the advantages of MMP when computing EELS maps. Since the MMP matrix is independent of the excitation, we can setup and factorize the matrix once and then efficiently solve the system for a large number of different sources. To compute the EELS maps shown in Fig. 9, we solved the system for 201 by 201 different beam positions. We find that both the resonance frequencies and the EELS maps are qualitatively similar to the results published in [22], but the resonance are slightly blue-shifted. Those deviations are readily explained by the omission of the silicon nitride membrane in our MMP simulations. 


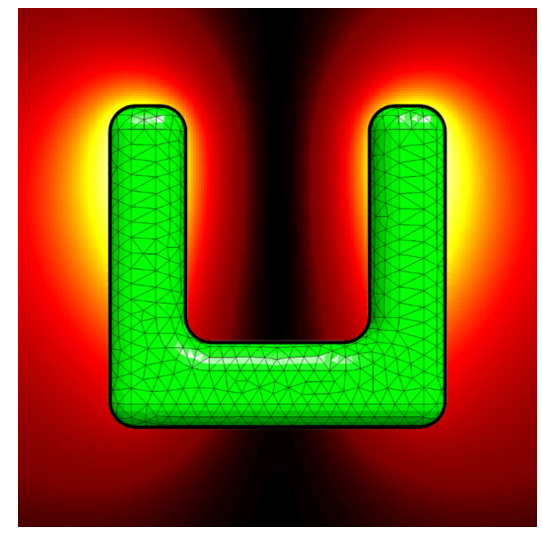

(a) $\lambda=1460 \mathrm{~nm}$

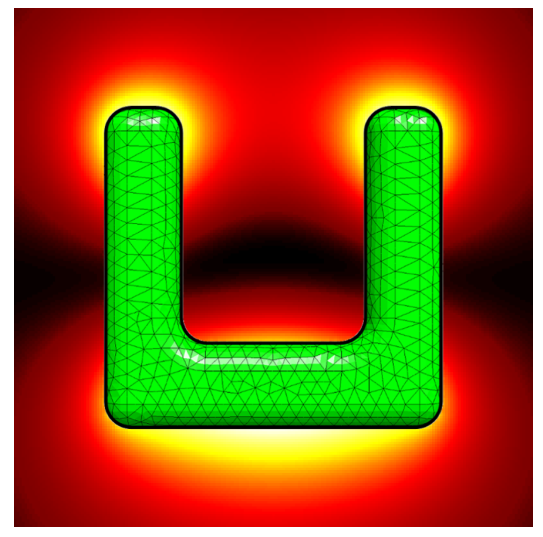

(b) $\lambda=845 \mathrm{~nm}$

Fig. 9 Normalized EELS maps for the first two modes of a plasmonic split-ring resonator.

\subsection{Electron Energy Loss Spectroscopy of a Plasmonic Disk-Dimer on a Membrane}

As discussed above, to obtain results comparable to experimental measurements, the inclusion of the substrate or membrane is essential. Here, we demonstrate that MMP can handle this by using the layered dipole expansions presented in Sect. 3.2. To this end, we model a plasmonic disk dimer made out of gold and deposited on a $30 \mathrm{~nm}$ thick silicon nitride membrane $\left(\varepsilon_{r}=4\right)$. The disk radius is $r=75 \mathrm{~nm}$ and its height is $t=30 \mathrm{~nm}$. The distance between the disks is taken to be $g=20 \mathrm{~nm}$. The geometry and the corresponding mesh are sketched in Fig. 10, where the dots indicate the positions of the electron beam excitation for EELS spectra computation. The resulting spectra are shown in Fig. 11.

We can clearly observe how the two different excitation positions lead to distinct resonance peaks. This can be explained by looking at the corresponding EELS maps depicted in Fig. 12.

Here, we see that the mode with a resonance at $773 \mathrm{~nm}$ shows very little signal at position $\# 1$. In contrast, the modes at $870 \mathrm{~nm}$ and $700 \mathrm{~nm}$ are comparatively weak at position \#2. To gain further insight into the nature of the different modes, we also use MMP to compute the distribution of the electric field in a plane slightly above the dimer. The results for the $z$-component (perpendicular to the cut plane) can be found in Fig. 13. These plots contain information about the relative phase, which allows us to identify the mode at $870 \mathrm{~nm}$ as the symmetric and the mode at $700 \mathrm{~nm}$ as the anti-symmetric combination of the fundamental mode of an individual disk (cf. [23]). Here, the higher-energetic mode at $700 \mathrm{~nm}$ corresponds to a dark mode and would not be visible when using classical optical spectroscopy. 


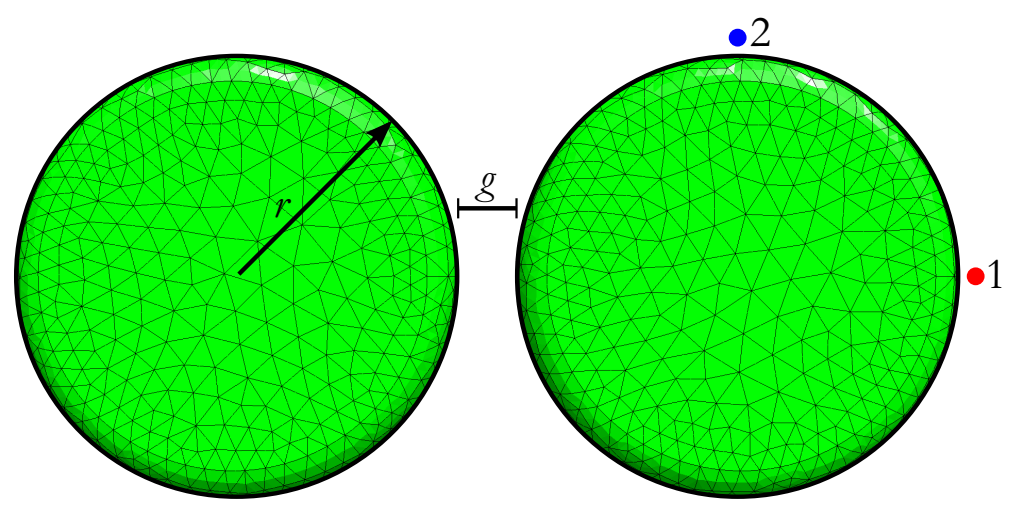

Fig. 10 Geometry and mesh for the disk-dimer experiment. The colored dots again indicate the positions of the electron beam excitation.

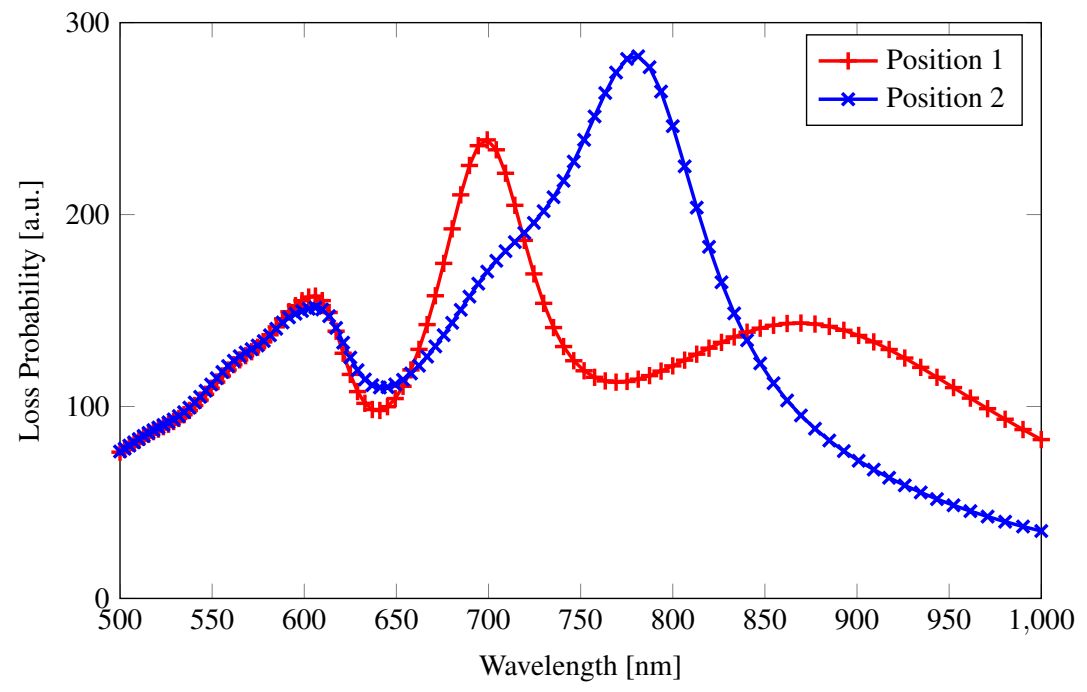

Fig. 11 Electron energy loss spectrum of a plasmonic disk-dimer calculated at two different electron beam positions.

\subsection{Comparison of MMP and DGTD for Electron Energy Loss Spectroscopy Calculations}

Finally, we compare the performance of our novel MMP implementation to an established Discontinuous Galerkin time-domain (DGTD) solver [24, 23, 25]. It is important to keep in mind that MMP is a frequency-domain method while DGTD works in the time-domain. For this comparison, we return to the EELS experiment with the golden split-ring resonator of Sect. 5.3 and compare the computation times of the two solvers for both EELS spectrum and EELS map computations. The sim- 


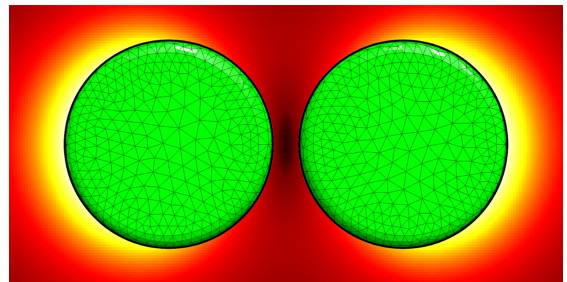

(a) $\lambda=870 \mathrm{~nm}$

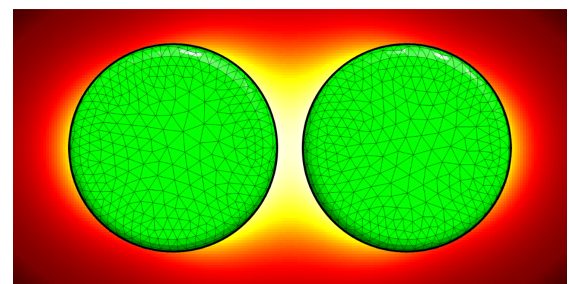

(b) $\lambda=700 \mathrm{~nm}$

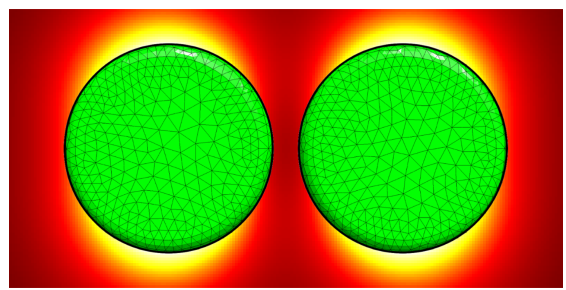

(c) $\lambda=773 \mathrm{~nm}$

Fig. 12 Normalized EELS maps for the first three modes of the plasmonic disk-dimer.

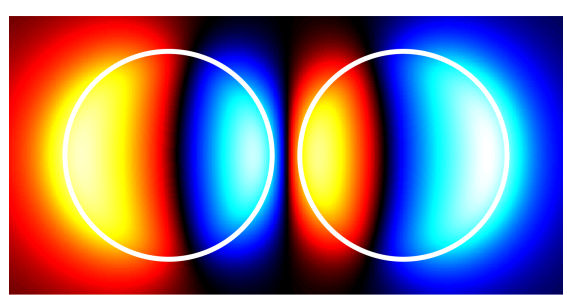

(a) $\lambda=870 \mathrm{~nm}$

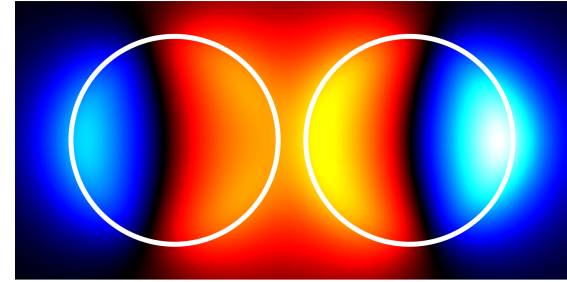

(b) $\lambda=700 \mathrm{~nm}$

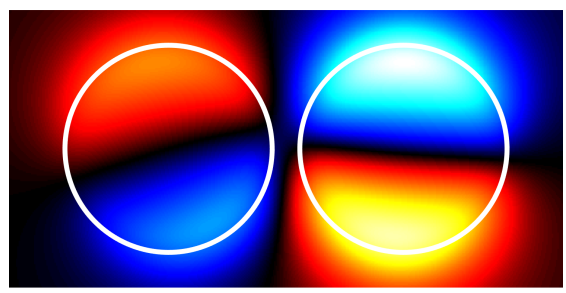

(c) $\lambda=773 \mathrm{~nm}$

Fig. 13 Normalized electric field in $z$-direction for the first three modes of a plasmonic disk-dimer. 
ulations were run on a desktop computer with Intel Xeon W3680 processor with 6 cores at $3.33 \mathrm{GHz}$ and $12 \mathrm{~GB}$ RAM. We restricted both solvers to run on a single core to get a fair comparison independent of the implementation of parallelization. To guarantee a similar accuracy of both methods, the same surface mesh was used for the split-ring resonator. For DGTD method, the given surface mesh was extended to a tetrahedral volume mesh using Netgen [10].

First, we computed the EELS spectrum and measured the time consumption of both methods. The timings of the individual steps performed in the MMP solver and the total time for DGTD are listed in Table 3.

Table 3 Computation times for EELS spectrum simulations with MMP or DGTD. In DGTD, the full spectrum is received in one simulation while in MMP it scales with the number of frequency points $n_{f}$.

\begin{tabular}{lrr}
\hline & MMP & DGTD \\
\hline Assembly (min) & $0.5 \cdot n_{f}$ & - \\
QR Decomposition (min) & $50.0 \cdot n_{f}$ & - \\
Solving Equation (min) & $<0.1 \cdot n_{f}$ & - \\
Loss Evaluation (min) & $<0.1 \cdot n_{f}$ & - \\
Total Time (min) & $50.6 \cdot n_{f}$ & 2505 \\
\hline
\end{tabular}

As a time-domain solver, DGTD computes the entire spectrum in a single run, while MMP requires a separate simulation per frequency point. Therefore, the time per frequency point in MMP needs to be multiplied by the number of points in a spectrum. Hence, for a good spectral resolution it is beneficial to use a time-domain solver while frequency-domain methods are only appropriate for a small number of frequencies. In our example, DGTD performs more efficiently once the number of frequency points exceeds 50 .

Second, the rasterization time for an EELS pattern was measured. Since MMP is a boundary discretization method in the frequency-domain, it can reuse the matrix decomposition for the different beam positions as they are just right-hand-sides. In contrast, DGTD requires a full calculation per point. While this does also deliver a full spectrum, maps are usually only required for very few selected frequencies. Hence, MMP easily outperforms DGTD for the computation of EELS maps. The corresponding timings can be found in Table 4.

Table 4 Computation times for EELS map simulations with MMP or DGTD. In DGTD, a full simulation is required for each point while in MMP the $\mathrm{QR}$ decomposition in the setup can be reused for all beam positions. Here, $n_{r}$ is the number of raster points.

\begin{tabular}{lrr}
\hline & MMP & DGTD \\
\hline Assembly (min) & 0.5 & - \\
QR Decomposition (min) & 50.0 & - \\
Solving \& Evaluation (min) & $0.06 \cdot n_{r}$ & - \\
Total Time (min) & $50.0+0.06 \cdot n_{r}$ & $2505 \cdot n_{r}$ \\
\hline
\end{tabular}


From this data, we find that both frequency and time-domain methods have their respective advantages. For the computationally more demanding EELS map computation, MMP is clearly preferable in our case. However, if we had used an example with substrate, the timings would have been more favorable for DGTD, since the evaluation of the Sommerfeld integrals is computationally expensive.

Finally, it should be noted that the absolute times of both methods can be improved significantly by parallelization. Simply using a parallel implementation of the matrix factorization and then distributing the different positions for an EELS map on multiple cores leads to an almost perfect scaling. Similarly, since each position corresponds to a separate computation, DGTD can also be expected to scale very well with the number of cores and processors. Additionally, as was shown in [23], the EELS computation with the DGTD method can be massively accelerated by using the computational power of GPUs.

\section{Summary and Outlook}

We presented a number of extensions to the well established multiple multipole program (MMP), which enhance the flexibility and usability of this technique. Our novel procedure to automatically generate a distribution of both expansions and matching points from a given surface mesh leads to a significant reduction in preparation time and allows to apply MMP to arbitrary geometries. In addition, the introduction of layered dipoles as expansions allows a more efficient simulation of realistic experiments where the nanostructures are usually deposited on substrates or membranes. Finally, with the introduction of an electron beam source and a suitable adaptive integration routine, MMP can also be used to compute electron energy loss spectra and EELS maps.

Overall, we showed that MMP is a well-tested, robust and fast simulation technique which is particularly well suited for the analysis of plasmonic nanostructures. Especially for the computation of EELS maps, the MMP method has strong advantages over alternative simulation techniques such as classical FEM or DGTD. Due to the small size of the system matrix, MMP allows to precompute and store a matrix decomposition. Then, even very large maps can be computed with relatively little computational effort. If, on the other hand, only the spectrum at a single point is required, MMP has some disadvantages over time-domain simulations.

As an outlook, we expect that a more sophisticated placement of the expansions based on the curvature of the surface could boost the efficiency enormously. Unfortunately, constructing an algorithm that reliably works for arbitrary geometries without sacrificing stability has proved difficult and further research is needed to achieve this goal. 


\section{References}

1. S.A. Maier, H.A. Atwater, Journal of Applied Physics 98(1), (2005)

2. F.J. García de Abajo, Reviews of Modern Physics 82(1), 209 (2010)

3. B.W. Reed, J.M. Chen, N.C. MacDonald, J. Silcox, G.F. Bertsch, Physical Review B 60, 5641 (1999)

4. F.J. García de Abajo, A. Howie, Physical Review B 65, 115418 (2002)

5. N. Geuquet, L. Henrard, Ultramicroscopy 110(8), 1075 (2010)

6. C. Matyssek, J. Niegemann, W. Hergert, K. Busch, Photonics and NanostructuresFundamentals and Applications 9(4), 367 (2011)

7. C. Hafner, Post-modern electromagnetics using intelligent MaXwell solvers (Wiley, Chichester, 1999)

8. C. Hafner, Numerische Berechnung elektromagnetischer Felder - Grundlagen, Methoden, Anwendungen. PhD Thesis, ETH Zurich, Zurich (1987)

9. C. Hafner. OpenMaXwell. URL http://openmax.ethz.ch/

10. J. Schöberl. Netgen Mesh Generator. URL http://sourceforge.net/projects/netgen-mesher/

11. W.C. Chew, Waves and fields in inhomogeneous media. IEEE Press series on electromagnetic waves (IEEE Press, New York, 1995)

12. G. Dural, M.I. Aksun, IEEE Transactions on Microwave Theory and Techniques 43(7), 1545 (1995)

13. A. Alparslan, Layered Media Green's Functions: Derivation and approximation techniques for all ranges and materials (LAMBERT Academic Publishing, Saarbrücken, 2011)

14. A. Alparslan, Numerical Analysis of Photonic Nano Structures in Layered Geometries. PhD Thesis, ETH Zurich, Zurich (2013)

15. R. Golubovic, A.G. Polimeridis, J.R. Mosig, IEEE Transactions on Antennas and Propagation 60(5), 2409 (2012)

16. M. Dogan, M.I. Aksun, A.K. Swan, B.B. Goldberg, M.S. Unlu, Journal of the Optical Society of America A - Optics, Image Science, and Vision 26(6), 1458 (2009)

17. L.F. Shampine, Journal of Computational and Applied Mathematics 211(2), 131 (2008)

18. D.E. Amos. Package for Bessel Functions of a Complex Argument and Nonnegative Order. URL http://www.netlib.org/amos/index.html

19. Tuxfamily. Eigen 3.1.2. URL http://eigen.tuxfamily.org/

20. Intel. Intel Math Kernel Library 11.0. URL http://software.intel.com/en-us/intel-mkl

21. Intel. Intel Threading Building Blocks 4.1. URL http://software.intel.com/en-us/intel-tbb

22. F. von Cube, S. Irsen, J. Niegemann, C. Matyssek, W. Hergert, K. Busch, S. Linden, Optical Materials Express 1(5), 1009 (2011)

23. F. von Cube, S. Irsen, R. Diehl, J. Niegemann, K. Busch, S. Linden, Nano letters 13(2), 703 (2013)

24. K. Busch, M. König, J. Niegemann, Laser \& Photonics Reviews 5(6), 773 (2011)

25. F. von Cube, J. Niegemann, S. Irsen, D.C. Bell, S. Linden, Physical Review B 89(11), 115434 (2014) 